\title{
PREVALÊNCIA DE INCAPACIDADE FUNCIONALE DEPENDÊNCIA EM IDOSOS ATENDIDOS EM UM CENTRO DE SAÚDE-ESCOLA DA UNIVERSIDADE DE SÃO PAULO
}

\author{
Jéssica Sponton Moura Minosso¹, Fernanda Amendola², Márcia Regina Martins Alvarenga ${ }^{3}$, Maria Amélia de Campos
}

Oliveira $^{4}$

\begin{abstract}
RESUMO: O conhecimento das condições de vida e saúde relacionadas à capacidade funcional de idosos pode subsidiar as proposições de intervenções para essa população. Foram objetivos do estudo descrever o perfil sociodemográfico de idosos atendidos em um serviço ambulatorial de geriatria, estimar a prevalência da incapacidade funcional e analisar as características a ela associadas. Estudo observacional, transversal, em que a população foi constituída por amostra aleatória de pessoas com idade igual ou superior a 60 anos atendidas em um centro de saúde-escola, localizado no município de São Paulo. A caracterização sócio-demográfica foi realizada mediante o uso de instrumento específico. A capacidade funcional foi avaliada por meio do Índice de Barthel; aplicou-se análise bivariada. Os idosos da faixa etária mais elevada apresentaram uma maior prevalência de incapacidade funcional. Conclui-se que a capacidade funcional do idoso é influenciada por seu perfil sociodemográfico e não somente por patologias e/ou deficiências.
\end{abstract}

PALAVRAS-CHAVE: Prevalência; Avaliação geriátrica; Assistência a idosos.

\section{PREVALENCE OF FUNCTIONAL DISABILITY AND DEPENDENCE IN OLDER ADULTS TREATED AT ASCHOOL-HEALTH CENTER OF THE SÃO PAULO UNIVERSITY}

\begin{abstract}
The knowledge of living and health conditions related to the elders' functional ability can support the propositions for interventions for this population group. The objectives were to describe the socio-demographic profile of elderly in a geriatrics ambulatory, to estimate the prevalence of functional disability and analyze the characteristics associated with it. It was an observational study, cross-sectional, in which the population was composed of a random sample of people aged equally or more than 60 years, treated at an academic affiliated health center located in São Paulo. The socio-demographic characteristics were accomplished by the use of a specific instrument. The Functional ability was assessed using the Barthel Index. Bivariate analysis was applied. As results, it was found that the elders of the higher age group had a higher prevalence of disability. The functional ability of the elderly is influenced by their sociodemographic profile and not only by diseases and / or disabilities.
\end{abstract}

KEYWORDS: Prevalence; Geriatric assessment; Old age assistance.

\section{PREVALENCIA DE LA INCAPACIDAD FUNCIONAL Y DEPENDENCIAEN ANCIANOS ATENDIDOS EN UN CENTRO DE SALUD-ESCUELA DE LA UNIVERSIDAD DE SÃO PAULO}

RESUMEN: El conocimiento de las condiciones de vida y salud relacionadas a la capacidad funcional de ancianos puede subsidiar las propuestas de intervenciones para esta población. Fueron objetivos del estudio describir el perfil sociodemográfico de los ancianos atendidos en un servicio ambulatorio de geriatría, estimar la prevalencia de incapacidad funcional y analizar las características asociadas a ella. Estudio observacional, transversal, en el cual la población fue constituida por muestra aleatoria de personas con edad igual o superior a 60 años atendidas en un centro de salud-escuela, ubicado en el municipio de San Paulo. La caracterización socio-demográfica fue realizada por medio del Índice de Barthel, se aplicó análisis bivariado. Los ancianos de la faja etaria más elevada presentaron una mayor prevalencia de incapacidad funcional. Se concluye que la capacidad funcional del anciano es influenciada por su perfil socio-demográfico y no sólo por patologías y/o deficiencias.

PALABRAS CLAVE: Prevalencia; Evaluación geriátrica; Asistencia a los ancianos.

${ }^{1}$ Enfermeira. Graduada pela Escola de Enfermagem da Universidade de São Paulo-EEUSP.

${ }^{2}$ Enfermeira do Programa Saúde da Família da Associação Congregação Santa Catarina. São Paulo, Brasil. Doutoranda pelo Programa de Pós-Graduação em Enfermagem da EEUSP.

${ }^{3}$ Enfermeira. Docente do Curso de Enfermagem da Universidade Estadual de Mato Grosso do Sul-UEMS.

${ }^{4}$ Enfermeira. Livre Docente. Professora Associada do Departamento de Enfermagem em Saúde Coletiva da EEUSP.

Autor correspondente:

Maria Amélia de Campos Oliveira

Escola de Enfermagem da Universidade de São Paulo

Av. Dr. Enéas de Carvalho Aguiar, 419 - 05403-000 - São Paulo-SP, Brasil Recebido: 30/06/09

E-mail: macampos@usp.br

Aprovado: 11/12/09

Cogitare Enferm 2010 Jan/Mar; 15(1):12-8 


\section{INTRODUÇÃO}

No Brasil, tal como em grande parte do mundo, observa-se o aumento na expectativa de vida da população, que passa por um processo de envelhecimento rápido e intenso ${ }^{(1)}$. Essa condição, denominada transição demográfica, pode ser explicada pela conexão de mecanismos que reduzem a mortalidade e aqueles que levam à diminuição da fecundidade geral. Com essa transição, o panorama de morbimortalidade da população altera-se profundamente, com diminuição da incidência das doenças infectocontagiosas, substituídas progressivamente por processos crônicos de desgaste e adoecimento.

Do ponto de vista individual, o aumento da longevidade ocasiona uma situação paradoxal: o desejo de viver cada vez mais coexiste com o medo de atingir essa meta com perda da autonomia e da independência ${ }^{(2)}$. No aspecto coletivo, os serviços de saúde têm como desafio responder às necessidades das pessoas com incapacidades e dependência, e de suas famílias. O sistema de saúde precisa ser capaz de identificar quais usuários apresentam maiores necessidades, a fim de implantar ações consoantes com as mesmas.

Na avaliação da qualidade de vida dos idosos, destaca-se o conceito de capacidade funcional, que reflete a habilidade de executar atividades que permitam cuidar de si próprio e viver de maneira independente. Assim como a autonomia, ou seja, a capacidade de se autodeterminar e executar os próprios desígnios, a capacidade funcional é considerada um elemento essencial da saúde.

Dentro dessa nova perspectiva, o envelhecimento saudável passa a ser entendido como resultante da interação multidimensional entre saúde física, saúde mental, estado emocional, independência nas atividades da vida diária, integração social, suporte familiar e independência econômica. Qualquer uma dessas dimensões, se comprometida, pode afetar a capacidade funcional do idoso ${ }^{(3)}$.

Estudos associam a diminuição da capacidade funcional do idoso à presença de doenças, deficiências ou problemas de saúde. Seus resultados também demonstram que a capacidade funcional é influenciada por condições demográficas, socioeconômicas, culturais e psicossociais ${ }^{(4,7)}$. O conhecimento das condições de vida e saúde relacionadas à incapacidade funcional de idosos pode subsidiar a proposição de intervenções para com essa população.
A Organização Mundial da Saúde recomenda que políticas de saúde na área de envelhecimento levem em consideração os determinantes e condicionantes da saúde ao longo de todo o curso de vida, o que inclui a situação social e econômica, mas também aspectos comportamentais, pessoais, culturais, além do ambiente físico e acesso a serviços de saúde. Recomenda ainda que tais estudos incidam sobre questões de gênero e desigualdades sociais, por sua potencial interferência nas condições de vida e saúde dos idosos ${ }^{(8)}$. Os objetivos do presente estudo foram: descrever o perfil sociodemográfico de idosos atendidos em um serviço ambulatorial, estimar a prevalência de incapacidade funcional e analisar as características a ela associadas.

\section{MATERIAL E MÉTODO}

Estudo observacional do tipo transversal. A população do estudo foi constituída por idosos com ou sem dependência, atendidos no Centro de Saúde Paula Souza da Faculdade de Saúde Pública da Universidade de São Paulo, da qual se obteve uma amostra aleatória de 100 pessoas com idade igual ou superior a 60 anos.

Considerou-se como critério de inclusão pessoas de ambos os sexos, com 60 anos de idade ou mais, cadastradas no Centro de Saúde Paula Souza, com ou sem algum grau de dependência e que concordassem em assinar o Termo de Consentimento Livre e Esclarecido.

A coleta de dados foi realizada no período de maio a setembro de 2007. Um questionário foi utilizado para os dados sociodemográficos e o Índice de Barthel aplicado como instrumento de avaliação da capacidade funcional.

O projeto foi previamente submetido à Comissão de Ética em Pesquisa da Escola de Enfermagem da USP (n-577/2006) bem como à direção do Centro de Saúde Paula Souza, obtendo aprovação de ambos. Todos os participantes foram informados sobre os objetivos da pesquisa e a confidencialidade dos dados, nos termos da Resolução 196/96, e assinaram o termo de consentimento livre e esclarecido.

Foram investigadas as seguintes variáveis sociodemográficas: sexo, idade, estado civil, número de pessoas na casa, renda mensal per capita em reais; renda familiar; escolaridade (em anos de estudo completos); atividade econômica (ativo ou inativo aposentado e/ou pensionista) e ocupação anterior à aposentadoria. Além disso, avaliou-se a condição 
funcional dos idosos como variável de desfecho.

\section{Índice de Barthel}

A avaliação da capacidade funcional recai sobre um conjunto de estados de saúde relacionados ao aumento de limitações funcionais, existindo diversas formas de mensurá-la. Inúmeros instrumentos foram desenvolvidos para este fim, entre eles o Índice de Barthel, utilizado neste estudo.

O Índice de Barthel pertence ao campo de avaliação de atividades da vida diária (AVDs) e mede o grau de assistência exigido por um indivíduo no cuidado pessoal, mobilidade, locomoção e eliminações. A independência funcional é avaliada por meio de 10 tarefas: alimentação, banho, vestuário, higiene pessoal, eliminações intestinais, eliminações vesicais, uso do vaso sanitário, passagem cadeira-cama, deambulação e escadas.

Cada item do instrumento é pontuado de acordo com o desempenho do paciente em realizar tarefas de forma independente, com alguma ajuda ou de forma dependente. Atribuindo-se os pontos em cada categoria, obtém-se uma pontuação geral, em função do tempo e da assistência necessária a cada paciente. Esta pontuação varia de 0 a 100, dividida em intervalos de cinco pontos, e os pontos de corte traduzem o grau de dependência dos indivíduos ${ }^{(9)}$.

Neste estudo foi utilizada a versão de Guimarães e Guimarães ${ }^{(10)}$, em língua portuguesa, já validada no Brasil. Em estudo anterior, o instrumento foi submetido a testes de reprodutibilidade e confiabilidade que mostraram níveis adequados para o valor total ${ }^{(11)}$

Alguns autores ${ }^{(9,12-14)}$ têm proposto diretrizes para a interpretação da pontuação do Índice de Barthel, sendo que a pontuação abaixo de 60 tem sido avaliada como indicativo de uma dependência mais severa. Neste estudo, optou-se por utilizar a classificação de Shah et al. ${ }^{(15)}$, que categoriza como "dependência total" um score de até 20 pontos, "dependência severa” de 21 a 60 pontos, "dependência moderada" de 61 a 90 pontos, “dependência leve” de 91 a 99 pontos e "independência” para os que atingem 100 pontos.

\section{Análise dos dados}

A análise dos dados foi feita mediante a apresentação descritiva dos resultados em frequência absoluta e relativa, média, desvio padrão, mediana e valores mínimos e máximos.

Realizou-se análise bivariada, utilizando o teste de hipóteses para diferenças entre médias ( $t$ de Student) para variáveis contínuas. Para verificar a associação entre duas variáveis categóricas foi usado o teste qui-quadrado $\left(\mathrm{c}^{2}\right)$ de Pearson ${ }^{(16)}$.

Como procedimento alternativo, nos casos em que ocorreu uma freqüência esperada menor que dois ou quando mais de $20 \%$ das freqüências esperadas foram menores que cinco, aplicou-se o teste exato de Fischer ${ }^{(16)}$, nas tabelas $2 \mathrm{X} 2$ e associação linear por linear em tabelas envolvendo mais de duas linhas ou colunas.

Todos os resultados foram analisados considerando-se o valor de $\mathrm{p}<0,05$ como diferença significativa ${ }^{(16)}$

\section{RESULTADOS}

\section{Caracterização sociodemográfica}

A aplicação do questionário sociodemográfico permitiu constatar que, da amostra de 100 idosos, $68 \%$ era do sexo feminino. A média de idade foi de 76,4 anos (dp =8,2) para os homens e 74,8 $(d p=6,8)$ para as mulheres. Em sua maioria, os idosos pertenciam à faixa etária de 70 a 79 anos (46\%), seguida da faixa de 80 ou mais anos (33\%) e de 6069 anos (21\%).

Em relação à escolaridade, $8 \%$ eram analfabetos, $37 \%$ tinham entre um e quatro anos de estudo, $16 \%$ entre cinco e $8,6 \%$ entre 9 e 11 anos e $31 \%$ com 12 ou mais anos de estudo. Os homens apresentaram escolaridade média de $8,9(\mathrm{dp}=5,7)$ anos de estudo e as mulheres a média de 6,9 (dp=5,1), porém esta última não foi significativamente menor $(\mathrm{t}=1,85 ; p=0,068)$.

Em relação à atividade laboral e ao tipo de benefício social recebido, verificou-se que os idosos eram aposentados em grande maioria. Entre as mulheres, notou-se uma menor quantidade de idosas aposentadas (60,3\%) que entre os homens (80,6\%). Em contrapartida, 23,5\% das mulheres eram pensionistas, enquanto que, entre os homens, essa porcentagem era de $3,2 \%$, apenas.

A renda familiar dos idosos entrevistados apresentou a seguinte distribuição: $18 \%$ recebiam um salário mínimo (SM) ou menos por mês, 12\%, entre um e dois SM e 57\%, mais que dois SM por mês. A renda per capita das famílias desses idosos estava assim distribuída: 34\% recebiam um SM ou menos por pessoa no mês, $23 \%$ entre um e dois SM e 30\%, 
mais de dois SM por pessoa por mês. Observou-se, portanto, que parte dos proventos dos idosos estava sendo usada para o sustento das demais pessoas com quem dividiam a moradia.

Quanto ao número de pessoas por família, verificou-se que $29 \%$ moravam sozinhos, $64 \%$ com uma a quatro pessoas e $7 \%$ dividiam a residência com cinco ou mais pessoas.

\section{Capacidade funcional}

Na atividade "alimentação", 93\% dos idosos eram independentes, cerca de $6 \%$ necessitavam de auxílio e apenas $1 \%$ era dependente. Para a "higiene pessoal”, 4\% dos idosos eram dependentes e, com relação ao "banho", 7\% eram dependentes, ou seja, não tomavam banho sozinhos ou requeriam assistência para lavar uma parte do corpo (pelo menos). Para "vestuário", 4\% eram dependentes e 5\% precisavam de auxílio.

Na função "eliminações intestinais", 3\% dos idosos eram incontinentes e $1 \%$ incontinente ocasional. Em relação à “eliminação vesical”, 13\% eram incontinentes e $11 \%$, incontinentes ocasionais. No uso do vaso sanitário, 3\% eram dependentes e $2 \%$ necessitavam de ajuda em alguma parte do processo. A tabela 1 destaca a média, o desvio-padrão, bem como os valores mínimos e máximos obtidos nos dez domínios analisados pelo Índice de Barthel.

Tabela 1 - Descrição do Índice de Barthel. São Paulo, 2008.

\begin{tabular}{lccc}
\hline Domínios & Pontuação & Média (dp) & Mín-max \\
\hline Alimentação & $0-10$ & $9,6(1,5)$ & $0-10$ \\
Banho & $0-05$ & $4,6(1,3)$ & $0-05$ \\
Vestuário & $0-10$ & $9,3(2,2)$ & $0-10$ \\
Higiene pessoal & $0-05$ & $4,8(1,0)$ & $0-05$ \\
$\begin{array}{l}\text { Eliminações } \\
\text { intestinais }\end{array}$ & $0-10$ & $9,6(1,8)$ & $0-10$ \\
$\begin{array}{l}\text { Eliminações } \\
\text { vesicais }\end{array}$ & $0-10$ & $8,1(3,5)$ & $0-10$ \\
Uso do vaso & $0-10$ & $9,6(1,8)$ & $0-10$ \\
sanitário & & & \\
$\begin{array}{l}\text { Passagem } \\
\text { cadeira-cama }\end{array}$ & $0-15$ & $14,4(2,3)$ & $0-15$ \\
$\begin{array}{l}\text { Deambulação } \\
\text { Escadas }\end{array}$ & $0-15$ & $13,9(2,8)$ & $0-15$ \\
ÍndiceTotal & $0-10$ & $8,7(3,0)$ & $0-10$ \\
\hline
\end{tabular}

Para "deambulação", 2\% eram dependentes, 1\% era independente no uso de cadeira de rodas e $14 \%$ necessitavam de ajuda mínima. Para "subir escadas", 8\% eram dependentes e 7\% necessitavam de ajuda. Para a categoria "passagem cadeira-cama", $1 \%$ era dependente, $2 \%$ necessitavam de grande ajuda e $5 \%$, de ajuda mínima.

A classificação geral dos idosos, segundo o grau de dependência - avaliado pelo Índice de Barthel, demonstrou que os entrevistados eram, em sua maioria, independentes. Os resultados estão descritos na tabela 2 .

Tabela 2 - Grau de dependência dos idosos segundo o Índice de Barthel. São Paulo, 2008.

\begin{tabular}{lcc}
\hline Grau de dependência & $\mathrm{N}$ & $\%$ \\
Dependência total & 2 & 2 \\
Dependência severa & 3 & 3 \\
Dependência moderada & 23 & 23 \\
Dependência leve & 9 & 9 \\
Independência & 63 & 63 \\
Total & 100 & 100 \\
\hline
\end{tabular}

A análise das associações entre fatores permitiu observar que apenas a faixa etária influenciou significativamente o grau de dependência, conforme mostra a tabela 3 .

\section{DISCUSSÃO}

A interação entre os aspectos sociodemográficos e ambientais e o estado de saúde física e mental influencia a capacidade funcional do idoso. Estudos americanos têm enfocado essa interação ${ }^{(17-19)}$.

Pesquisas anteriores evidenciaram a associação entre o sexo feminino e dependência funcional, o que não foi confirmado neste estudo. Esta associação, muitas vezes, é explicada levando-se em consideração a maior longevidade das mulheres em comparação aos homens e, consequentemente, o risco maior para o desenvolvimento de doenças incapacitantes ${ }^{(6,20)}$. No estudo de Bambuí(21), tanto o sexo masculino como o feminino apresentaram proporções semelhantes de necessidade de cuidados para realizar pelo menos uma das atividades de vida diária.

Os idosos da faixa etária mais elevada apresentaram maior prevalência de incapacidade funcional. Em geral, limitações funcionais são mais frequentes em indivíduos mais velhos e estudos têm 
Tabela 3 - Distribuição dos idosos independentes ou com algum grau de dependência segundo variáveis socioeconômicas e demográficas. São Paulo, 2008.

\begin{tabular}{|c|c|c|c|c|c|}
\hline Variável & Categoria & Independência & $\begin{array}{l}\text { Algum grau de } \\
\text { dependência }\end{array}$ & Total & $\mathrm{p}$ \\
\hline \multirow[t]{2}{*}{ Sexo } & Masculino & 22 & 10 & 32 & 0,414 \\
\hline & Feminino & 41 & 27 & 68 & \\
\hline \multirow[t]{3}{*}{ Faixa Etária } & 60 a 69 & 15 & 06 & 21 & $0,011^{*}$ \\
\hline & 70 a 79 & 34 & 12 & 46 & \\
\hline & 80 e mais & 14 & 19 & 33 & \\
\hline \multirow[t]{3}{*}{ Renda per capita } & $<1 \mathrm{SM}$ & 20 & 14 & 34 & 0,217 \\
\hline & $\geq 1 \mathrm{e} \leq 2 \mathrm{SM}$ & 13 & 10 & 23 & \\
\hline & $>2 \mathrm{SM}$ & 23 & 7 & 30 & \\
\hline \multirow[t]{4}{*}{ Escolaridade } & Analfabeto & 07 & 04 & 11 & 0,757 \\
\hline & 1 a 4 anos & 22 & 14 & 36 & \\
\hline & 5 a 8 anos & 09 & 06 & 15 & \\
\hline & 9 anos e mais & 25 & 13 & 38 & \\
\hline \multirow[t]{4}{*}{ Estado civil } & Solteiro & 13 & 4 & 17 & 0,869 \\
\hline & Casado & 22 & 13 & 35 & \\
\hline & Viúvo & 17 & 17 & 34 & \\
\hline & Separado & 11 & 2 & 13 & \\
\hline
\end{tabular}

*Associação estatisticamente significativa para $\mathrm{p}<0,050$

mostrado que, entre idosos, o risco de incapacidade funcional aumenta consideravelmente com o passar dos anos.

Estudo epidemiológico desenvolvido em 2000, no município de São Paulo, envolvendo 2.143 idosos, mostrou que $80,7 \%$ dos entrevistados não apresentavam limitações funcionais que impediam o autocuidado. Entretanto, entre os idosos com idade avançada (75 anos e mais), existia uma queda no desempenho funcional e consequente aumento no grau de limitações funcionais. Entre as atividades que apresentavam maior grau de dependência, tanto para o sexo feminino quanto o masculino, estavam: vestir-se, mobilizar-se (deitar e levantar-se da cama) e banhar-se ${ }^{(20)}$.

Na presente investigação, embora a renda per capita não tenha mostrado associação significativa com a dependência, como ocorreu em alguns estudos $^{(4,20)}$, sua relevância não pode ser desprezada.
Também é necessário ressaltar que os idosos participantes da presente pesquisa vivem em uma região do município de São Paulo com baixo índice de necessidades de saúde ${ }^{(22)}$.

\section{CONCLUSÃO}

Este estudo descreveu características sociodemográficas de idosos atendidos em um serviço ambulatorial de um centro de saúde-escola da Universidade de São Paulo, relacionando-as com a incapacidade funcional.

As características identificadas neste e em outros estudos, e que se associaram à dependência, ajudam a identificar uma rede causal associada ao declínio da capacidade funcional. No envelhecimento, a interação de múltiplos potenciais de desgaste, relacionados com a saúde física, mental, inserção 
econômica e social, entre outros, pode levar à incapacidade e, eventualmente, à dependência.

As ações preventivas voltadas especificamente para tais determinantes ou condicionantes podem proporcionar benefícios para a manutenção ou melhoria da funcionalidade nesse grupo populacional.

$\mathrm{Na}$ área da Enfermagem, o foco de atenção ao idoso carece de ampliação. Certamente, as intervenções de enfermagem serão mais efetivas à medida que seu campo de atuação torne-se mais abrangente, de modo a levar em conta os aspectos sociodemográficos envolvidos no cuidado à saúde. Orientações para a manutenção da capacidade funcional e a prevenção de incapacidades nem sempre coincidem com a realidade social das populações, daí a importância dos estudos desenvolvidos com o propósito de identificar os aspectos relacionados ao declínio da autonomia e da independência.

\section{REFERÊNCIAS}

1. Ramos LR. Epidemiologia do envelhecimento. In: Freitas EV, Cançado FAX, Gorzoni ML, Neri AL, Py L, Rocha SM, organizadores. Tratado de geriatria e gerontologia. Rio de Janeiro: SBGG; Guanabara Koogan, 2002.p.72-8

2. Paschoal SMP, Jacob Filho W, Litvoc J. Development of elderly quality of life index - EQOLI: theoreticalconceptual framework, chosen methodology, and relevant items generation. Clinics. 2007;62(3):279-88.

3. Parahyba MI, Veras R. Diferenciais sociodemográficos no declínio funcional em mobilidade física entre os idosos no Brasil. Ciênc Saúde Coletiva. 2008;13(4):1257-64.

4. Rosa TEC, Benício MHD, Latorre MRDO, Ramos LR. Fatores determinantes da capacidade funcional entre idosos. Rev Saúde Pública. 2003;37(1):40-8.

5. Giacomin KC, Peixoto SV, Uchoa E, Lima-Costa MF. Estudo de base populacional dos fatores associados à incapacidade funcional entre idosos na Região Metropolitana de Belo Horizonte, Minas Gerais, Brasil. Cad Saúde Pública. 2008;24(6):1260-70.

6. Santos KA, Koszuoski R, Dias-da-Costa JS; Pattussi MP. Factors associated with functional incapacity among the elderly in Guatambu, Santa Catarina State, Brazil. Cad Saúde Pública. 2007;23(11):2781-8.

7. Lopes MA, Hototian SR, Bustamante SEZ, Azevedo D, Tatsch M, Bazzarella MC et al. Prevalence of cognitive and functional impairment in a community sample from
São Paulo, Brazil. Dement Geriatr Cogn Disord 2008;25:135-43.

8. Paskulin LMG. Fatores associados à qualidade de vida de idosos de um distrito sanitário de Porto Alegre/RS. [tese]. São Paulo (SP): Universidade Federal de São Paulo; 2008.

9. Mc Dowel I, Newell C. Measuring health: a guide to rating scales and questionaires. 2nd ed. New York: Oxford University Press; 1996.

10. Guimarães RB, Guimarães RB. Validação e adaptação cultural para a língua portuguesa de escalas de avaliação funcional em doenças cerebrovasculares: uma tentativa de padronização e melhora da qualidade de vida. Rev Bras Neurol. 2004;40(3):5-13.

11. Minosso JSM, Oliveira MAC, Amendola F, Alvarenga MRM. Validação do Índice de Barthel para idosos atendidos em ambulatórios no Brasil. Acta Paul Enferm. No prelo 2009.

12. Mahoney FI, Barthel DW. Functional evaluation: the Barthel Index. Md State Med J. 1965;14:61-5.

13. Gresham GE, Philips TF. Labi MLC. ADL status in stroke: relative merits of three standard indexes. Arch Phys Med Rehab. 1980;61:355-8.

14. Cid-Ruzafa J, Damián-Moreno J. Valoración de la discapacidad física: el Indice de Barthel. Rev Esp Salud Pública. 1997;71:127-37.

15. Shah S, Vanclay F, Cooper B. Improving the sensitivity of the Barthel Index for stroke rehabilitation. J Clin Epidemiol. 1989;42:703-9.

16. Dawson B, Trapp RG. Bioestatística básica e clínica. $3^{\mathrm{a}}$ ed. São Paulo: McGraw-Hill; 2003

17. Kington RS, Smith JP. Socioeconomic status and racial and ethnic differences infunctional status associated with chronic diseases. Am J Public Health. 1997;87:805-10.

18. Roos NP, Havens B. Predictors of successful aging: a twelve-year study of Manitoba elderly. Am J Public Health. 1991;81(1):63-8.

19. Guralnik JM, LaCroix AZ, Abbott RD, Berkman LF, Satterfield S, Evans DA, Wallace RB. Maintaining mobility in late life. Demographic characteristics and chronic conditions. Am J Epidemiol. 1993;137:845-57.

20. Ramos LR. Fatores determinantes do envelhecimento 
saudável em idosos residentes em centro urbano: Projeto Epidoso, São Paulo. Cad Saúde Pública. 2003;19:793-8.

21. Giacomin KC, Uchoa E, Firmo JOA, Lima-Costa MF. Projeto Bambuí: um estudo de base populacional da prevalência e dos fatores associados à necessidade de cuidador entre idosos. Cad Saúde Pública. 2005;21(1):80-91.

22. Duarte YAO. Desempenho funcional e demandas assistenciais. In: Lebrão ML, Duarte YAO. SABE Saúde Bem-estar e Envelhecimento: o Projeto SABE no município de São Paulo: uma abordagem inicial. Brasília: Organização Pan-Americana da Saúde; 2003. p.183-200. 